\title{
Autoimmune Optic Neuritis in the Common Marmoset Monkey: Comparison of Visual Evoked Potentials with MRI and Histopathology
}

\author{
Ricarda Diem, ${ }^{1}$ Iris Demmer, ${ }^{2}$ Susann Boretius, ${ }^{3}$ Doron Merkler, ${ }^{4}$ Barthel Schmelting, \\ Sarab K. Williams, ${ }^{1}$ Muriel B. Sättler, ${ }^{2}$ Matbias Bäbr, ${ }^{2,6}$ Thomas Michaelis, ${ }^{3}$ Jens Frabm, ${ }^{3}$ \\ Wolfgang Brück, ${ }^{4,6}$ and Eberbard Fuchs ${ }^{2,5,6}$
}

Purpose. To assess the use of visual evoked potentials (VEPs) for the in vivo detection of impaired visual function in a marmoset model of multiple sclerosis. The sensitivity of the VEP recordings was determined by comparison with magnetic resonance imaging (MRI) and histopathology.

Methods. Baseline VEPs were recorded in six healthy marmoset monkeys in response to light-flash stimulation. Experimental autoimmune encephalomyelitis (EAE) was induced in four of the six monkeys. Clinical scores were assessed daily, and VEPs were recorded every second week. In vivo MRI and subsequent histopathology of the brains and optic nerves were performed at the end of the study.

Results. After induction of EAE, all four marmosets exhibited clinical signs between day 26 and 38 after immunization. VEPs were normal during the induction phase of the disease, but deteriorated in amplitude with the occurrence of clinical symptoms in all animals. MRI revealed bilateral optic neuritis and signal alterations in the optic tracts and occipital subcortical white matter in two of the animals. In the remaining two animals, MRI detected signal alterations in the occipital subcortical white matter. Histopathologic results were concordant with the MRI findings.

Conclusions. VEPs are an easily accessible noninvasive tool for measuring visual function and diagnosing impairment of the visual pathway in a marmoset EAE model. (Invest Ophthalmol Vis Sci. 2008;49:3707-3714) DOI:10.1167/iovs.081896

From the ${ }^{1}$ Neurologische Klinik der Universität des Saarlandes, Homburg/Saar, Germany; ${ }^{2}$ Abteilung Neurologie and ${ }^{4}$ Abteilung Neuropathologie, Georg-August-Universität Göttingen, Göttingen, Germany; ${ }^{3}$ Biomedizinische NMR Forschungs $\mathrm{GmbH}$ am Max-Planck-Institut für Biophysikalische Chemie, Göttingen, Germany; ${ }^{5}$ Klinische Neurobiologie, Deutsches Primatenzentrum, Göttingen, Germany; and ${ }^{6}$ Institut für Multiple-Sklerose-Forschung, Universitätsmedizin der GeorgAugust-Universität und Gemeinnützige Hertie-Stiftung, Göttingen, Germany.

Supported by the Gemeinnützige Hertie-Stiftung and the Medical Faculty of the University of Göttingen (Junior Research Group Program to $\mathrm{RD}$ ).

Submitted for publication February 17, 2008; revised April 2, 2008; accepted June 17, 2008.

Disclosure: R. Diem, None; I. Demmer, None; S. Boretius, None; D. Merkler, None; B. Schmelting, None; S.K. Williams, None; M.B. Sättler, None; M. Bähr, None; T. Michaelis, None; J. Frahm, None; W. Brück, None; E. Fuchs, None

The publication costs of this article were defrayed in part by page charge payment. This article must therefore be marked "advertisement" in accordance with 18 U.S.C. $\$ 1734$ solely to indicate this fact.

Corresponding author: Ricarda Diem, Neurologische Klinik der Universität des Saarlandes, Kirrberger Straße, Gebäude 90, 66421 Homburg/Saar, Germany; ricarda.diem@uniklinikum-saarland.de.
$\mathbf{M}$ ultiple sclerosis (MS) is a chronic autoimmune disease of the central nervous system (CNS). For many decades, inflammation and demyelination have been considered to be the predominant histopathologic features. More recently, histopathologic as well as magnetic resonance spectroscopy and imaging studies have revealed that axonal damage is a further important pathologic aspect of MS and is the underlying cause of chronic disability in patients. ${ }^{1-6}$

Optic neuritis is one of the most common and frequently the first clinical manifestation of MS. The likelihood of the development of MS 10 years after isolated optic neuritis is up to $56 \%$ and depends on the frequency of additional white matter lesions in the brain. ${ }^{7}$ Optic neuritis is mainly characterized by a subacute loss of vision. Visual acuity after an episode of optic neuritis can recover within a few weeks, but this recovery remains incomplete in approximately one third of patients. ${ }^{8}$ In addition, most patients report persistent visual disturbances and show optic nerve atrophy detectable by MRI. ${ }^{9,10}$

Animal models of MS that mimic diverse aspects of the disease have been established in different species by using various agents and modes of immunization. ${ }^{11-14}$ In this respect, experimental autoimmune encephalomyelitis (EAE) induced by myelin oligodendrocyte glycoprotein (MOG) in the common marmoset monkey (Callitbrix jacchus) is an important model of MS, ${ }^{15,16}$ as its pathophysiology involves both T-cell and antibody responses against several myelin antigens. Thus, this lesion type closely resembles the pattern II type of active MS lesions, which is the most prominent type in chronic MS. ${ }^{17,18}$ Furthermore, acute lesions in this EAE model appear to be identical with the acute and actively demyelinating lesions observed in human MS $^{19}$ and are frequently located along the primary visual pathway. ${ }^{20,21}$

Visual evoked potentials (VEPs) have been used as a noninvasive tool for the in vivo investigation of the visual pathway in rodent models of EAE. ${ }^{22,23}$ In MOG-induced autoimmune optic neuritis in rats, we have used VEPs to monitor the disease course and to test the effects of different therapeutic agents. ${ }^{23-26}$ In these studies, a strong correlation between VEP results and histopathologic data was obtained. ${ }^{23,25}$ Although there are a few VEP studies of Old World monkeys, ${ }^{27-30}$ less information is available concerning VEPs in New World monkeys, such as the common marmoset. In anesthetized marmosets, VEPs were recorded during a pharmacologic study. ${ }^{31}$ However, systematic data describing variations in VEP peak latencies or amplitudes in marmosets are not yet available.

To investigate the affection of the primary visual pathway during the course of EAE in marmoset monkeys, we established VEP recordings in healthy animals by determining representative peak latencies and amplitudes. Subsequently, 
Table 1. Animal Data and Study Outline

\begin{tabular}{clrccc}
\hline Animal & Sex & $\begin{array}{c}\text { Age } \\
\text { (mo) }\end{array}$ & $\begin{array}{c}\text { Body } \\
\text { Weight } \\
\text { (g) }\end{array}$ & $\begin{array}{c}\text { VEP (Days before } \\
\text { and after } \\
\text { Immunization) }\end{array}$ & $\begin{array}{c}\text { MRI } \\
\text { (d) }\end{array}$ \\
\hline A & Male & 36 & 382 & $-7 ; 0 ; 15 ; 26$ & 26 \\
B & Male & 46 & 370 & $-7 ; 0 ; 15 ; 26 ; 40$ & 40 \\
C & Female & 57 & 408 & $-12 ; 0 ; 15 ; 28$ & 28 \\
D & Male & 104 & 478 & $-12 ; 0 ; 30 ; 41$ & 41 \\
E & Male & 57 & 502 & $*$ & \\
F & Male & 40 & 431 & & \\
\hline
\end{tabular}

Sex, age, and body weight of individual marmoset monkeys as well as the timing of VEP and MRI recordings.

* Animals $\mathrm{E}$ and $\mathrm{F}$ were not immunized.

we recorded VEPs in animals with MOG-induced EAE and compared the results with the disability score, MRI-detectable lesions, and histopathologic assessments.

\section{Methods}

\section{Animals}

Six adult common marmoset monkeys were examined in this study (Table 1). The animals were obtained from the marmoset colony at the German Primate Center (Göttingen, Germany). They were housed in pairs on a regular day-night cycle at $26^{\circ} \mathrm{C}$ in $55 \%$ relative humidity, with free access to food and water. Animal experiments were conducted in accordance with the European Communities Council Directive of November 24, 1986 (86/ EEC), with the National Institutes of Health Guide for the Care and Use of Laboratory Animals, and with the ARVO Statement for the Use of Animals in Ophthalmic and Vision Research. The number of animals used in our study and the length of the in vivo observation period were approved by the Government of Lower Saxony, Germany.

\section{Study Outline}

To obtain baseline VEP data, we performed two recording sessions in each healthy animal: the first within 12 days before immunization and the second immediately before immunization (a third follow-up measurement was obtained in animals $\mathrm{E}$ and $\mathrm{F} 2$ weeks after the second one). Follow-up VEP measurements in animals A to D were started at day 15 after immunization and were subsequently repeated every second week. After each animal developed clinical signs corresponding to a disability score of at least 3 , the final VEP recording was performed and was followed by magnetic resonance imaging (MRI). Immediately afterward, the animals were killed and the brains, optic nerves, and spinal cords were processed for histopathologic analysis. Table 1 summarizes details of the animals and the study outline.

\section{VEP Recordings}

For VEP recordings, the monkeys were anesthetized by intramuscular injection of $9 \mathrm{mg} / \mathrm{mL}$ alfaxalone and $3 \mathrm{mg} / \mathrm{mL}$ alfadolone (Saffan; $1.5 \mathrm{~mL} / \mathrm{kg}$; Schering Plough Animal Health, Omaha, NE), 0.25 mg/kg diazepam (Valium; Roche, Mannheim, Germany), and $0.025 \mathrm{mg} / \mathrm{kg}$; glycopyrronium bromide (Robinul, Cerner Multum, Denver, CO). Each VEP session lasted 30 to 60 minutes. When the reflexes were strongly reduced, the animals were secured in a chairlike holder and fixed with elastic tapes. Stainless-steal needle electrodes were passed intracutaneously to a position $10 \mathrm{~mm}$ above the inion and directly over the vertex. The reference electrode was placed intracutaneously in the right thigh. For visual stimulation of the healthy animals (baseline measurements), light flashes of 20- $\mu$ s duration were provided at temporal frequencies of 1 as well as $6 \mathrm{~Hz}$. To reduce the measurement time after disease induction, we used only 6-Hz stimulation during the follow-up measurements, as this stimulation mode produced the most reproducible results.

Both eyes in each animal were measured separately. The goggles (LS-101J; Nihon Kohden, Tokyo, Japan) were placed in front of the stimulated eye with a distance of approximately $5 \mathrm{~mm}$ and a constant mean luminance of $15 \mathrm{~cd} / \mathrm{m}^{2}$. During the measurements, the nonstimulated eye was covered by a patch. After the flash stimulation, signals were registered for $250 \mathrm{~ms}$, amplified, band-pass filtered between 0.5 and $70 \mathrm{~Hz}$, and averaged across 60 stimulations (C2X; Sigma Medizin-Technik GmbH, Thum, Germany). The VEP recordings were evaluated in terms of latencies of peaks $\mathrm{N} 1, \mathrm{P} 2$, and N2. The amplitude was defined as the difference between N1 and P2 $(\mathrm{A}=\mathrm{N} 1$ - P2). Data were analyzed from three different measurements in each animal at the different time points.

\section{Induction and Evaluation of EAE}

For EAE induction, recombinant rat (rr)MOG corresponding to the $\mathrm{N}$-terminal sequence of rat MOG (amino acids 1-125) was expressed in Escherichia coli and purified to homogeneity, as previously described. ${ }^{32}$ The purified protein was dissolved in $6 \mathrm{M}$ urea and dialyzed against $20 \mathrm{mM}$ sodium acetate buffer ( $\mathrm{pH} 3.0)$, to obtain a soluble preparation that was stored at $-20^{\circ} \mathrm{C}$.

The immunization procedure was performed as previously described. ${ }^{21,33}$ In brief, before immunization, animals were anesthetized by intramuscular injection of alfaxalone-alfadolone, diazepam, and glycopyrronium bromide as described earlier. Each animal received two posterior thoracic and two posterior sacral subcutaneous injections of a total amount of $100 \mu \mathrm{L}$ homogenate containing $50 \mu \mathrm{g}$ rrMOG emulsified in complete Freund's adjuvant (CFA; Difco, Detroit, MI).

The course of the disease was monitored daily by trained and experienced observers using a disability score first reported by Villoslada et al. ${ }^{34}$ and further developed in our laboratory as described by Boretius et al. ${ }^{33}$ The scores for the following parameters were added: loss of body weight $(0 / 1 / 2)$, spontaneous movement $(0 / 1 / 2 / 3)$, eyelid reflex $(0 / 1 / 2)$, paresis $(0 / 1 / 2 / 3)$, and grip strength $(0 / 1 / 2 / 3)$. The numbers in brackets indicate the severity with 0 as no effect.

\section{Magnetic Resonance Imaging}

For MRI, we used a previously established protocol. ${ }^{33}$ Anesthesia was started by intramuscular injections, as described earlier. Subsequently, the animals were intubated with a self-made polyethylene endotracheal tube (inner/outer diameter $=1.3 / 2.2 \mathrm{~mm}$ ) and kept under inhalation anesthesia $\left(0.4 \%-1.0 \%\right.$ isoflurane in $70: 30 \mathrm{~N}_{2} \mathrm{O}-$ $\mathrm{O}_{2}$ ). Respiration was monitored by a signal derived from a homemade pressure transducer fixed to the animal's chest. Pulse rate and oxygen saturation were measured with a pulse oximeter (Nonin Medical, Plymouth, MN). The animals were held prone by a homemade acrylic stereotaxic device for marmoset monkeys, including ear and eye bars and a plate holder.

MRI was performed at $2.35 \mathrm{~T}$ with an $4.7 \mathrm{~T} / 400-\mathrm{mm}$ magnet (MRBR; Magnex, Scientific, Abington, UK) equipped with a scanner (DBX system; Bruker Biospin, Ettlingen, Germany). For signal detection, an elliptical surface coil $(40 \times 45 \mathrm{~mm})$ adapted to the head of the monkey was used and combined with a Helmholtz coil (100-mm diameter) for signal excitation. In each animal, $\mathrm{T}_{2^{-}}$ weighted MRI (3-D fast spin-echo sequence; TR/TE, 3000/97 ms; 16 echoes; echo spacing, $12.3 \mathrm{~ms}$; and measurement time, 29 minutes) was performed at $330 \mu \mathrm{m}$ isotropic spatial resolution covering a $42.24 \times 42.24 \times 42.24-\mathrm{mm}$ field of view.

\section{Histopathology}

Immediately after MRI, animals received a lethal dose of xylazine/ketamine. Brains with optic nerves and optic bulbs attached were dissected 
$1 \mathrm{~Hz}$

A

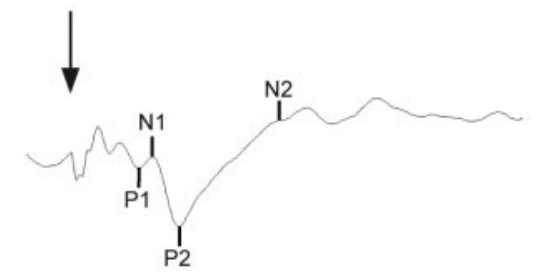

B

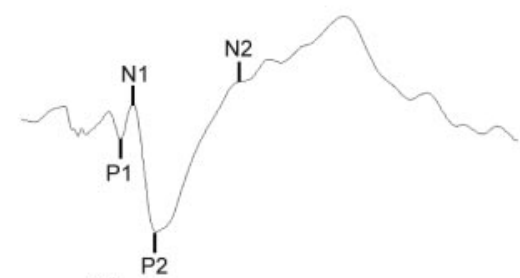

C
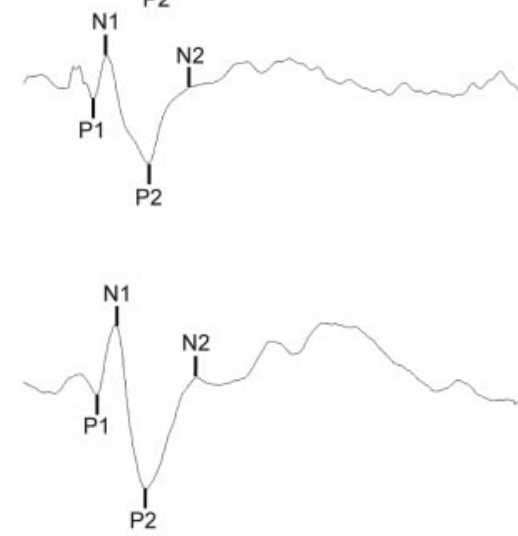

FigURE 1. Representative examples of VEPs in response to light-flash stimulation at (left) 1 and (right) $6 \mathrm{~Hz}$ in six healthy marmosets (A-F). $A r$ rows: light-flash stimuli. VEPs comprised a first (N1) and second (N2) negative peak as well as a first (P1) and second (P2) positive peak as indicated. During sham stimulation, recordings were performed without application of the flash light stimulus.

E

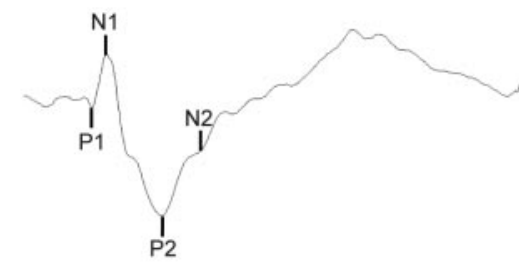

F

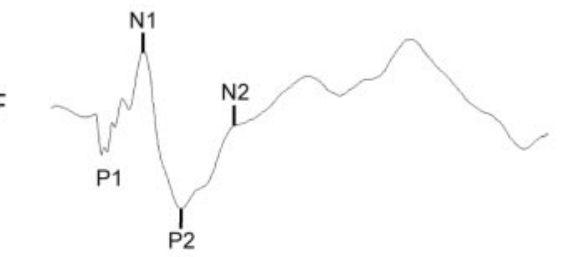

$10 \mu \mathrm{V}$

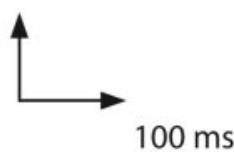

$6 \mathrm{~Hz}$

A

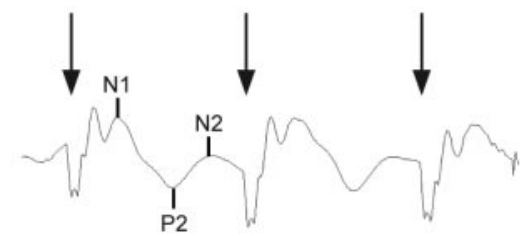

B

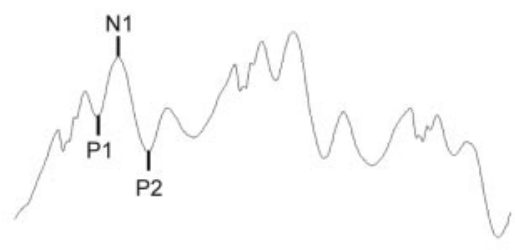

C

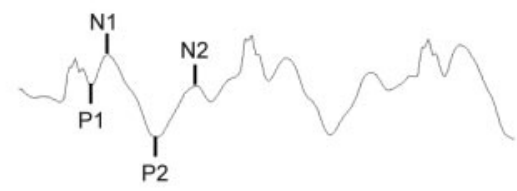

D

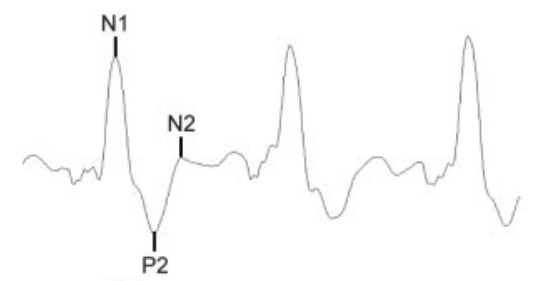

E

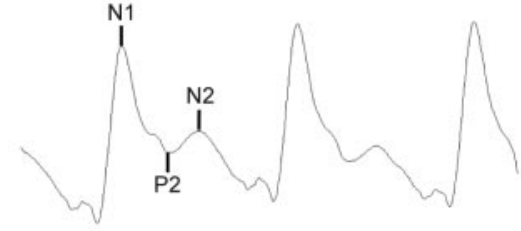

and split sagittally. The right half and the spinal cord were immersion fixed with 4\% $0.1 \mathrm{M}$ sodium phosphate-buffered paraformaldehyde $(\mathrm{pH}$ 7.4) for 78 hours and subsequently processed for paraffin embedding. The other half of the brain was snap frozen in liquid nitrogen.
Histologic evaluation was performed on 3 - $\mu$ m-thick sections stained with hematoxylin-eosin (HE), Luxol fast blue/Periodic acid shift (LFB/PAS), and Bielschowsky silver impregnation, to assess inflammation, demyelination, and axonal pathology, respectively. 


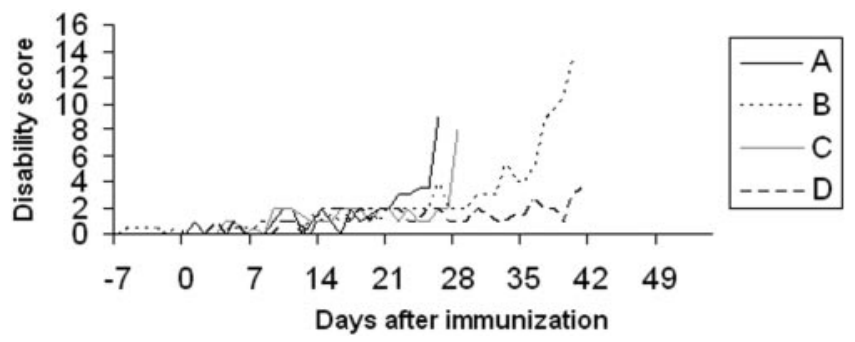

FIGURE 2. Disability scores after MOG-immunization in four marmosets (A-D). The neurologic score added the following parameters: loss of body weight $(0 / 1 / 2)$, spontaneous movement $(0 / 1 / 2 / 3)$, eye lid reflex $(0 / 1 / 2)$, paresis $(0 / 1 / 2 / 3)$, and grip strength $(0 / 1 / 2 / 3)$.

Histologic sections of optic nerves, optic chiasm, optic tract, and subcortical white matter of the visual cortex were analyzed for the presence or absence of demyelinating inflammatory lesions.

\section{Results}

\section{VEPs in Healthy Marmosets}

VEPs were recorded in all six healthy marmosets (Fig. 1). Although there was some variation between the VEP curves of individual animals, the potentials were stable over the different measurements, with a slightly higher reproducibility of the 6-Hz data. VEPs seemed not to be influenced by repeated anesthesia. The most consistent peak was the first negative peak (N1) with a mean latency of $44 \mathrm{~ms}$ after flash stimulation with $1 \mathrm{~Hz}$ and a mean latency of $47 \mathrm{~ms}$ after flash stimulation with $6 \mathrm{~Hz}$. This peak was often but not consistently preceded by a small positive peak (P1). A second positive peak (P2) varied interindividually within a range of 75 to 112 ms (mean: 85 ms after flash stimulation with $1 \mathrm{~Hz}$ and $89 \mathrm{~ms}$ after stimulation with $6 \mathrm{~Hz}$ ). A second negative peak (N2) had its mean at $112 \mathrm{~ms}$ after a $1-\mathrm{Hz}$ flash stimulation and $122 \mathrm{~ms}$ after a $6-\mathrm{Hz}$ flash stimulation. During some measurements, there were also later peaks, but they appeared to be not as consistent as the peaks just described. The mean peak-to-peak-amplitudes were 12.5 and $12.3 \mu \mathrm{V}$ for flash stimulation of 1 and $6 \mathrm{~Hz}$, respectively.

\section{Disability Scores of Animals after Immunization with MOG}

The time of onset and the severity of symptoms showed a certain degree of variability between individuals. Figure 2 gives the neurologic scores for animals A-D over the whole duration of the experiment. Values above 2 indicate the clinical onset of EAE, which occurred at days 26 (animal A), 34 (B), 28 (C), and 38 (D). During the course of the experiment, none of the animals showed a remission of symptoms. The experiments were stopped according to the individual disability score.

\section{VEPs of Animals after Immunization with MOG}

At day 15 after induction of EAE, the animals produced VEPs with peak latencies and amplitudes comparable to baseline (Figs. 1, 3). In animal $\mathrm{D}$, this measurement could not be performed due to problems with the anesthesia.

At day 26 and 28 after immunization, the respective VEPs of animals $A$ and $C$ revealed a generalized and marked reduction in peak-to-peak amplitudes to less than $4 \mu \mathrm{V}$ (except for the right eye of animal $\mathrm{C}$, in which a potential could no longer be detected). Animals B and D presented with a slower disease progression and still had normal VEPs at the time of the second follow-up at days 26 and 30 after immunization, respectively. At the time of the third follow-up measurement in animal B, only N1 was present. In animal $D$, no VEPs could be recorded at this time point.

Quantitative analyses of VEPs in response to $6 \mathrm{~Hz}$ stimulation before and after immunization of animals A-D are summarized in Table 2. Pronounced amplitude reductions or complete loss of potentials were confirmed in animals A (day 26), B (day 40), C (day 28), and D (day 41).

\section{MRI of Animals with MOG-Induced EAE}

In each animal, $\mathrm{T}_{2}$-weighted images revealed hyperintensities in white-matter structures of the visual system including the optic nerves (with additional swelling), chiasma, optical tract, and occipital subcortical areas. This is illustrated in Figure 4 which compares $T_{2}$-weighted images of affected areas with those of normal tissue. The size and location of the lesions in the visual system varied between animals. Animals A and D presented with optic neuritis and lesions in the entire visual pathway, whereas the other two animals were affected in the optic tract (animal C) and occipital subcortical areas (animals B and $\mathrm{C}$ ). These results are summarized in Table 3.

\section{Histopathology of Animals with MOG-Induced EAE}

Histopathologic analysis was performed on the optic pathway of the different animals. Myelin stained with Luxol fast blue and periodic acid shift (LFB/PAS) revealed perivascularly accentuated but also large confluent demyelinated lesions within the analyzed brain regions. In general, most of the plaques were early active with few inactive lesions, according to previously established criteria. ${ }^{21,35}$ The active plaques within the occipital subcortical white matter showed a sharp demarcation between the lesion and the periplaque white matter (Fig. 5). Histologic analysis of optic nerves showed a variable degree of demyelination. In some cases, inflammation and demyelination were incomplete, comprising only a fraction of the cross-sectional area (Fig. 5 ), whereas in other cases, cross sections of optic nerves revealed complete demyelination. In general, axonal structures were relatively preserved within the demyelinated lesions.

\section{Comparison of VEPs with MRI and Histopathology}

Bilateral optic nerve lesions were detected by MRI in animals $\mathrm{A}$ and $\mathrm{D}$ at a time when VEPs could no longer be observed or showed a severe reduction in amplitude. Histopathology of the optic nerves of these animals confirmed severe bilateral optic neuritis characterized by demyelination, inflammation, and axonal loss. In animals B and C, MRI did not indicate optic neuritis, although the histopathologic analysis revealed a slight inflammatory infiltration of the optic nerves. However, VEPs in these animals were severely flattened, and some peaks were no longer present. This may be explained either by the partial infiltration of the optic nerves as indicated by histopathology or by the large whitematter lesions in the occipital subcortical regions detected by both MRI and histopathology. The direct comparison of MRI and histopathologic data is given in Table 3.

\section{Discussion}

In this study, we initially performed VEP recordings in healthy marmosets to define peak latencies and amplitudes 

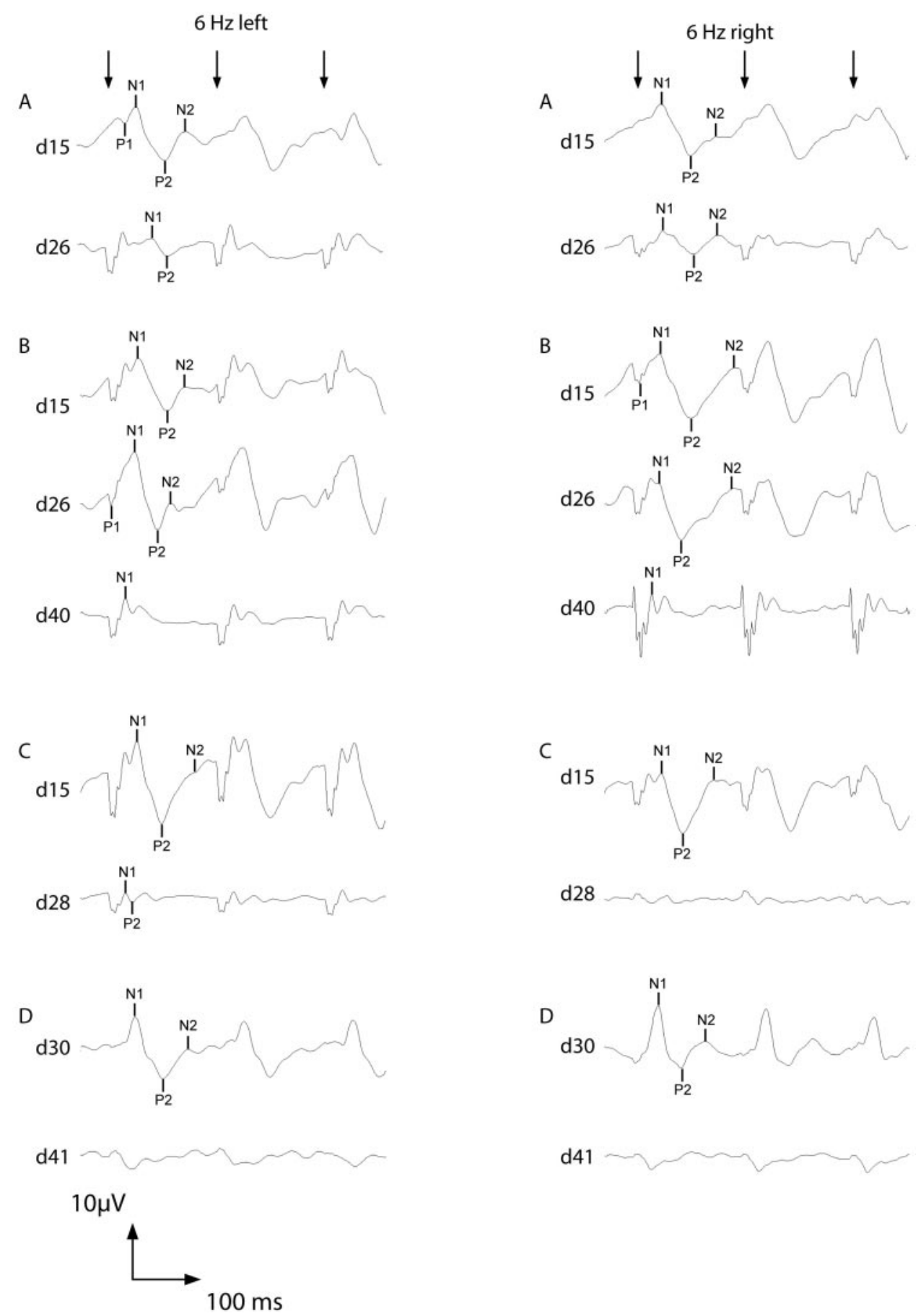

FIGURE 3. Representative examples of VEPs in response to light-flash stimulation at $6 \mathrm{~Hz}$ (arrows) of both eyes in four marmosets after MOGimmunization (A-D). 
Table 2. Visual Evoked Potentials in Response to 6-Hz Flashlight Stimuli

\begin{tabular}{|c|c|c|c|c|}
\hline & N 1 (ms) & P 2 (ms) & N 2 (ms) & Amplitude $(\mu V)$ \\
\hline \multicolumn{5}{|l|}{ A Left } \\
\hline d-7 & $47.8 \pm 2.9$ & $92.8 \pm 0.8$ & $120.4 \pm 5.7$ & $10.7 \pm 0.2$ \\
\hline do & $51.6 \pm 1.7$ & $94.1 \pm 4.1$ & $122.1 \pm 1.2$ & $9.1 \pm 1.3$ \\
\hline d15 & $49.7 \pm 0.5$ & $95.7 \pm 1.9$ & $125.6 \pm 4.0$ & $9.6 \pm 0.9$ \\
\hline$d 26$ & $60.8 \pm 10.1$ & $91.2 \pm 3.8$ & ND & $3.2 \pm 0.5$ \\
\hline \multicolumn{5}{|c|}{ A Right } \\
\hline d-7 & $43.0 \pm 6.3$ & $91.3 \pm 4.8$ & $121.7 \pm 6.5$ & $8.4 \pm 2.3$ \\
\hline do & $53.0 \pm 5.0$ & $104.8 \pm 5.0$ & $136.9 \pm 2.7$ & $7.1 \pm 1.6$ \\
\hline d15 & $41.7 \pm 5.4$ & $94.7 \pm 5.4$ & $118.4 \pm 1.3$ & $10.4 \pm 0.2$ \\
\hline d26 & $52.5 \pm 16.4$ & $92.5 \pm 16.9$ & $118.2 \pm 39.8$ & $3.7 \pm 0.9$ \\
\hline \multicolumn{5}{|l|}{ B Left } \\
\hline $\mathrm{d}-7$ & $39.7 \pm 0.8$ & $74.9 \pm 7.3$ & $107.9 \pm 1.3$ & $11.4 \pm 0.8$ \\
\hline do & $42.9 \pm 2.1$ & $77.5 \pm 2.4$ & $110.8 \pm 0.4$ & $9.7 \pm 1.3$ \\
\hline d15 & $46.1 \pm 0.9$ & $89.0 \pm 1.9$ & $116.5 \pm 0.7$ & $8.5 \pm 1.0$ \\
\hline $\mathrm{d} 26$ & $38.5 \pm 1.0$ & $75.8 \pm 1.9$ & $99.5 \pm 0.7$ & $15.2 \pm 0.4$ \\
\hline d40 & $48.6 \pm 1.3$ & ND & ND & ND \\
\hline \multicolumn{5}{|c|}{ B Right } \\
\hline d-7 & $43.8 \pm 2.0$ & $81.8 \pm 7.1$ & $111.1 \pm 4.7$ & $12.1 \pm 2.1$ \\
\hline do & $53.4 \pm 1.9$ & $79.1 \pm 6.0$ & $108.8 \pm 4.1$ & $10.1 \pm 2.9$ \\
\hline d15 & $40.4 \pm 1.4$ & $87.1 \pm 4.3$ & $117.4 \pm 3.1$ & $12.7 \pm 3.4$ \\
\hline d26 & $39.1 \pm 2.0$ & $80.4 \pm 5.2$ & $120.3 \pm 6.1$ & $11.5 \pm 1.6$ \\
\hline $\mathrm{d} 40$ & $48.0 \pm 1.0$ & ND & ND & ND \\
\hline \multicolumn{5}{|l|}{ C Left } \\
\hline $\mathrm{d}-12$ & $45.3 \pm 2.0$ & $75.4 \pm 1.0$ & $118.8 \pm 5.3$ & $11.4 \pm 0.3$ \\
\hline do & $43.7 \pm 1.9$ & $77.9 \pm 3.1$ & $116.0 \pm 4.9$ & $9.8 \pm 1.1$ \\
\hline d15 & $41.9 \pm 1.0$ & $81.9 \pm 2.8$ & $121.0 \pm 4.0$ & $10.8 \pm 1.0$ \\
\hline $\mathrm{d} 28$ & $55.9 \pm 1.3$ & $73.9 \pm 2.7$ & ND & $1.4 \pm 0.1$ \\
\hline \multicolumn{5}{|c|}{ C Right } \\
\hline $\mathrm{d}-12$ & $42.3 \pm 1.0$ & $82.1 \pm 5.5$ & $119.9 \pm 3.6$ & $14.5 \pm 3.1$ \\
\hline do & $44.4 \pm 5.0$ & $95.0 \pm 11.0$ & $127.2 \pm 2.5$ & $9.6 \pm 1.4$ \\
\hline d15 & $40 \pm 2.2$ & $78.5 \pm 0.5$ & $113.6 \pm 6.7$ & $26.4 \pm 1.2$ \\
\hline $\mathrm{d} 28$ & ND & ND & ND & ND \\
\hline \multicolumn{5}{|l|}{ D Left } \\
\hline $\mathrm{d}-12$ & $39.1 \pm 3.3$ & $82.9 \pm 4.9$ & $125.1 \pm 6.9$ & $15.9 \pm 3.1$ \\
\hline do & $40.5 \pm 4.1$ & $77.4 \pm 2.2$ & $120.0 \pm 4.7$ & $13.0 \pm 0.3$ \\
\hline d30 & $38.1 \pm 1.1$ & $83.7 \pm 1.4$ & $127.6 \pm 5.3$ & $11.1 \pm 0.6$ \\
\hline $\mathrm{d} 41$ & ND & ND & ND & ND \\
\hline \multicolumn{5}{|l|}{ D Right } \\
\hline $\mathrm{d}-12$ & $38.3 \pm 1.0$ & $81.1 \pm 5.7$ & $111.9 \pm 0.7$ & $16.4 \pm 0.4$ \\
\hline do & $40.8 \pm 0.9$ & $80.7 \pm 4.9$ & $114.4 \pm 4.8$ & $18.2 \pm 2.4$ \\
\hline d30 & $35.6 \pm 1.9$ & $76.4 \pm 1.3$ & $113.5 \pm 1.3$ & $10.2 \pm 2.0$ \\
\hline d41 & ND & ND & ND & ND \\
\hline
\end{tabular}

Peak latencies and peak-to-peak amplitudes (mean $\pm \mathrm{SD}, n=3$ ) for animals A-D. d, day before or after immunization with MOG; ND, not detectable.

shown to suppress VEPs extensively, in response to pattern and flash light stimulation. ${ }^{29,37}$

During the course of our study, the reduction in amplitude correlated with the clinical onset of EAE in each individual animal. In contrast to other species, all monkeys had normal VEPs during the induction of EAE until days 26 to 41 after immunization. In rats, VEPs gradually deteriorated after disease induction beginning with slight disturbances as early as 1 week before the clinical manifestation of symptoms. ${ }^{25}$ In a guinea pig model of EAE, it was also demonstrated that the delay in VEP latency precedes the clinical impairment. ${ }^{38}$ These differences may reflect a delayed but subsequently more acute disease development in marmosets.

Another important difference between monkey and rodent EAE is that the distribution of lesions is much more heterogeneous in marmosets. Whereas nearly $100 \%$ of brown Norway rats with pathologic VEP responses after MOG-immunization showed bilateral optic nerve inflamma- tion, ${ }^{23,25}$ only two of four marmosets in our study had severe optic neuritis, as detected by MRI and histopathology. In the other two marmosets, extensive lesions within the subcortical parts of the occipital lobe may explain the severe deterioration in the VEPs at the respective time points. The heterogeneous distribution of inflammatory demyelinating lesions, together with the variability of the clinical disease course and the onset of symptoms in our study, is in good agreement with previous studies of MOG-induced EAE in marmosets (for a review, see Ref. 39).

In summary, we have demonstrated that VEPs can be recorded in marmosets and provide a sensitive tool for monitoring the affection of the visual pathway in models of chronic inflammatory CNS diseases. As nonhuman primates share a high degree of immunologic similarity with humans, EAE models could be helpful during the preclinical development of new therapeutic options. In this context, it is of particular importance to establish noninvasive and sensitive methods for monitoring drug effects on disease progression in vivo.
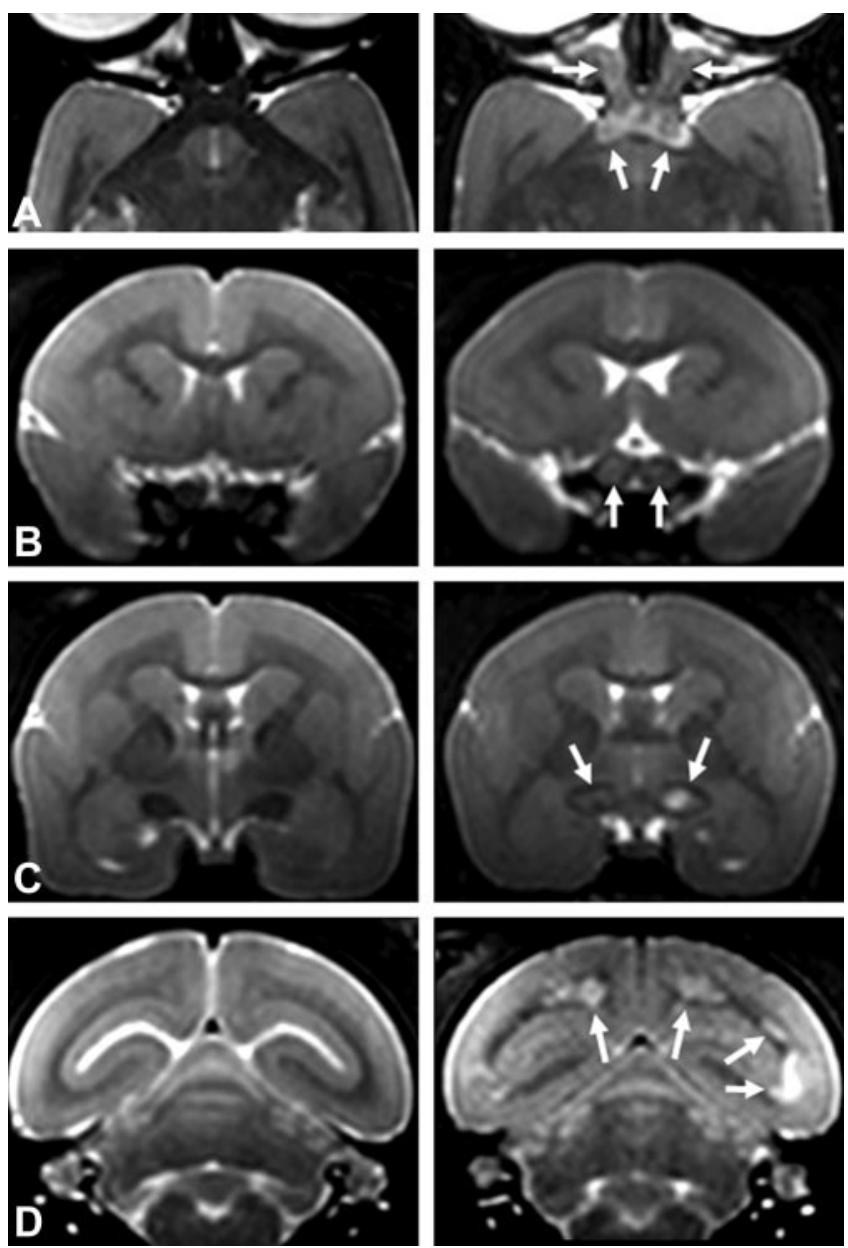

FIGURE 4. $\mathrm{T}_{2}$-weighted MRI of (left) normal and (right) affected tissue after MOG-immunization in (A-C) animal D (day 41) and (D) animal B (day 40). (A) Transverse sections parallel to the optic nerve, chiasm, and optic tract, as well as (B) the coronal sections perpendicular to the optic nerve and $(\mathbf{C})$ the optic tract demonstrated inflamed regions of the optic nerves (arrows) which appeared hyperintense and swollen. (D) Posterior coronal sections depict white-matter lesions (arrows) in the left and right occipital cortices. 
TABLe 3. MRI Abnormalities within the Primary Visual Pathway and Correlation with Histopathologic Results

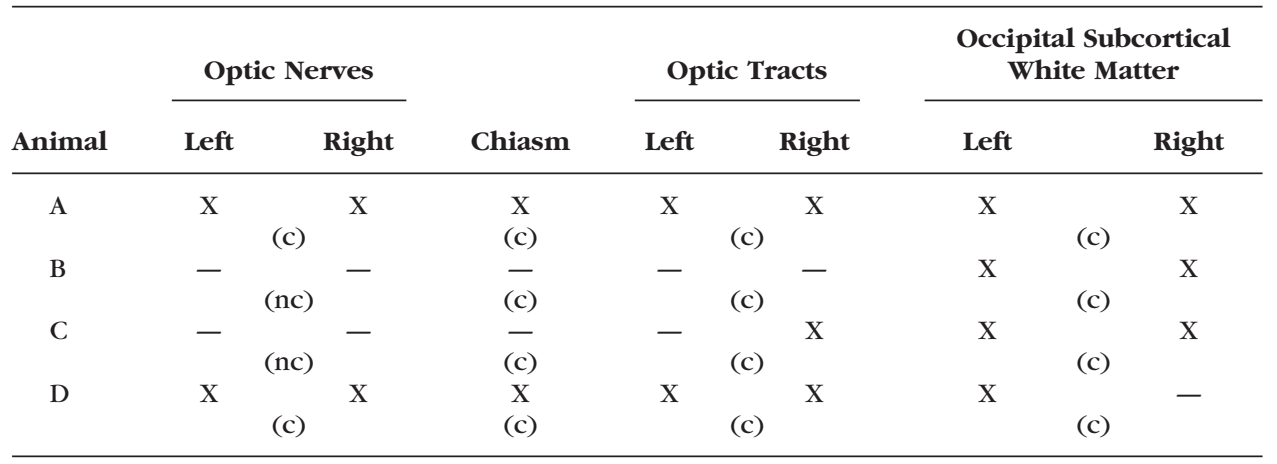

Hyperintensities in T2-weighted MRIs of the optic nerves, optic tracts, and occipital subcortical white matter. X, abnormal; - , normal; c, existing correlation with histopathology (inflammatory demyelinating lesions were found by histopathology in the areas that appeared hyperintense); nc, no clear correlation with histopathologic findings (MRI did not indicate lesions that were detected by histopathology).
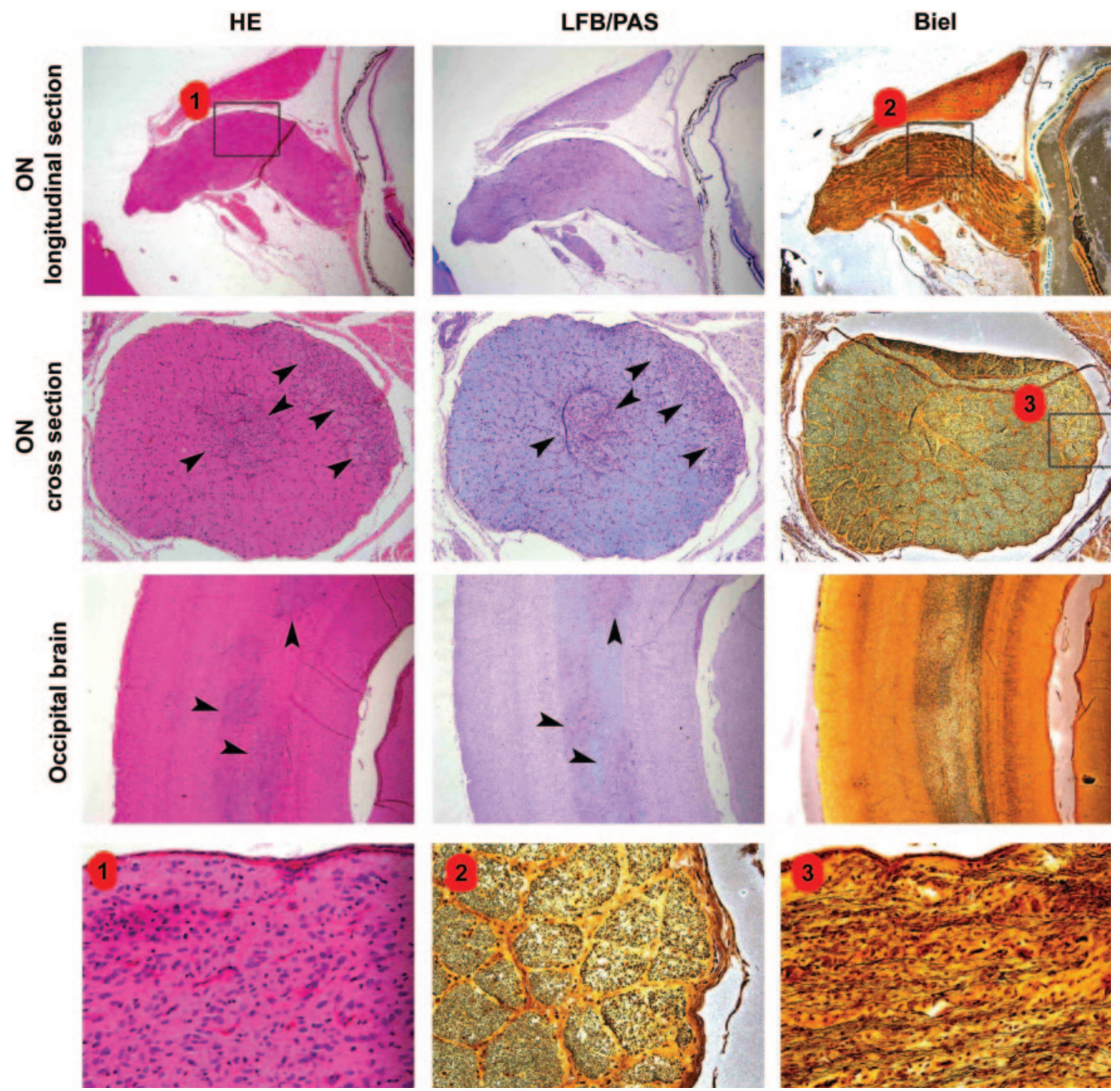

FigURE 5. Histopathologic analyses of optic nerves and occipital subcortical white matter. Representative images of (left, inflammation) HE-, (middle, demyelination) LFB/PAS-, and (right, axons) Bielschowsky-stained sections are shown. EAE lesions were detected on longitudinal sections (top line) and cross sections (middle line) of optic nerves. Dense inflammatory infiltrates were evident on HE-stained sections within demyelinated areas of the optic nerves (inset 1). Note that axonal structures were largely preserved within demyelinated areas (insets 2 and 3 ). Subcortical white matter of occipital brain regions (third row) showed perivascularly accentuated demyelinated lesions (arrowheads). 


\section{Acknowledgments}

The authors thank Cornelia Heckmann for excellent technical assistance.

\section{References}

1. Davie CA, Barker GJ, Webb S, et al. Persistent functional deficit in multiple sclerosis and autosomal dominant cerebellar ataxia is associated with axon loss. Brain. 1995;118:1583-1592.

2. Davie CA, Hawkins CP, Barker GJ, et al. Serial proton magnetic resonance spectroscopy in acute multiple sclerosis lesions. Brain. $1994 ; 117: 49-58$

3. Ferguson B, Matyszak MK, Esiri MM, et al. Axonal damage in acute multiple sclerosis lesions. Brain. 1997;120:393-399.

4. Losseff NA, Webb SL, O'Riordan JI, et al. Spinal cord atrophy and disability in multiple sclerosis: a new reproducible and sensitive MRI method with potential to monitor disease progression. Brain. 1996;119:701-708.

5. Trapp BD, Peterson J, Ransohoff RM, et al. Axonal transection in the lesions of multiple sclerosis. N Engl J Med. 1998;338:278-285.

6. Trapp BD, Ransohoff R, Rudick R. Axonal pathology in multiple sclerosis: relationship to neurologic disability. Curr Opin Neurol. 1999;12:295-302.

7. Beck RW, Trobe JD, Moke PS, et al. High- and low-risk profiles for the development of multiple sclerosis within 10 years after optic neuritis: experience of the optic neuritis treatment trial. Arch Opbthalmol. 2003;121:944-949.

8. Beck RW, Gal RL, Bhatti MT, et al. Visual function more than 10 years after optic neuritis: experience of the optic neuritis treatment trial. Am J Opbthalmol. 2004;137:77-83.

9. Hickman SJ, Brierley CM, Brex PA, et al. Continuing optic nerve atrophy following optic neuritis: a serial MRI study. Mult Scler. 2002;8:339-342

10. Hickman SJ, Kapoor R, Jones SJ, et al. Corticosteroids do not prevent optic nerve atrophy following optic neuritis. $J$ Neurol Neurosurg Psychiatry. 2003;74:1139-1141

11. Kornek B, Storch MK, Weissert R, et al. Multiple sclerosis and chronic autoimmune encephalomyelitis: a comparative quantitative study of axonal injury in active, inactive, and remyelinated lesions. Am J Patbol. 2000;157:267-276.

12. Storch MK, Stefferl A, Brehm U, et al. Autoimmunity to myelin oligodendrocyte glycoprotein in rats mimics the spectrum of multiple sclerosis pathology. Brain Patbol. 1998;8:681-694.

13. Weissert R, Wallstrom E, Storch MK, et al. MHC haplotype-dependent regulation of MOG-induced EAE in rats. J Clin Invest. 1998; 102:1265-1273.

14. Wekerle H, Kojima K, Lannes-Vieira J, et al. Animal models. Ann Neurol. 1994;36(suppl):S47-S53.

15. Massacesi L, Genain CP, Lee-Parritz D, et al. Active and passively induced experimental autoimmune encephalomyelitis in common marmosets: a new model for multiple sclerosis. Ann Neurol. 1995; 37:519-530.

16. t'Hart BA, van Meurs M, Brok HPM, et al. A new primate model for multiple sclerosis in the common marmoset. Immunol Today. 2000;21:290-297.

17. Lassmann $H$. The pathology of multiple sclerosis and its evolution. Pbilos Trans R Soc Lond B Biol Sci. 1999;354:1635-1640.

18. Lucchinetti C, Bruck W, Parisi J, et al. A quantitative analysis of oligodendrocytes in multiple sclerosis lesions: a study of 113 cases. Brain. 1999;122:2279-2295.

19. Genain CP, Hauser SL. Experimental allergic encephalomyelitis in the New World monkey Callithrix jacchus. Immunol Rev. 2001; 183:159-172.
20. Boretius S, Demmer I, Diem R, et al. MRI visualization of MOGinduced optic neuritis in a rat model of EAE. Proc Intl Soc Mag Reson Med. 2004;11:1451.

21. Merkler D, Schmelting B, Czeh B, et al. Myelin oligodendrocyte glycoprotein-induced experimental autoimmune encephalomyelitis in the common marmoset reflects the immunopathology of pattern II multiple sclerosis lesions. Mult Scler. 2006;12:369-374.

22. Bilbool N, Kaitz M, Feinsod M, et al. Visual evoked potentials in experimental allergic encephalomyelitis. J Neurol Sci. 1983;60: 105-115.

23. Meyer R, Weissert R, Diem R, et al. Acute neuronal apoptosis in a rat model of multiple sclerosis. J Neurosci. 2001;21:6214-6220.

24. Diem R, Sattler MB, Merkler D, et al. Combined therapy with methylprednisolone and erythropoietin in a model of multiple sclerosis. Brain. 2005;128:375-385.

25. Hobom M, Storch MK, Weissert R, et al. Mechanisms and time course of neuronal degeneration in experimental autoimmune encephalomyelitis. Brain Patbol. 2004;14:148-157.

26. Sättler MB, Merkler D, Maier K, et al. Neuroprotective effects and intracellular signaling pathways of erythropoietin in a rat model of multiple sclerosis. Cell Death Differ. 2004;11(suppl 2):S181-S192.

27. Ghilardi MF, Marx MS, Onofrj MC, et al. Scalp distribution of pattern visual evoked potentials in normal and hemianopic monkeys. Physiol Behav. 1987;41:297-302.

28. Hayreh SS, Masarani RM, Yamada T, et al. Experimental allergic encephalomyelitis. I. Optic nerve and central nervous system manifestations. Invest Ophthalmol Vis Sci. 1981;21:25-69.

29. Kulikowski JJ, Robson AG, Murray IJ. Scalp VEPs and intra-cortical responses to chromatic and achromatic stimuli in primates. Doc Ophthalmol. 2002;105:243-279.

30. Schroeder CE, Tenke CE, Givre SJ, et al. Striate cortical contribution to the surface-recorded pattern-reversal VEP in the alert monkey. Vision Res. 1991;31:1143-1157.

31. Philippens IH, Vanwersch RA, Groen B, et al. Subchronic physostigmine pretreatment in marmosets: absence of side effects and effectiveness against soman poisoning with negligible postintoxication incapacitation. Toxicol Sci. 2000;53:84-91.

32. Adelmann M, Wood J, Benzel I, et al. The N-terminal domain of the myelin oligodendrocyte glycoprotein (MOG) induces acute demyelinating experimental autoimmune encephalomyelitis in the Lewis rat. J Neuroimmunol. 1995;63:17-27.

33. Boretius S, Schmelting B, Watanabe T, et al. Monitoring of EAE onset and progression in the common marmoset monkey by sequential high-resolution 3D MRI. NMR Biomed. 2005;18:1-9.

34. Villoslada P, Hauser SL, Bartke I, et al. Human nerve growth factor protects common marmosets against autoimmune encephalomyelitis by switching the balance of $\mathrm{T}$ helper cell type 1 and 2 cytokines within the central nervous system. J Exp Med. 2000;191: 1799-1806

35. Lucchinetti C, Brück W, Parisi J, et al. Heterogeneity of multiple sclerosis lesions: implications for the pathogenesis of demyelination. Ann Neurol. 2000;47:707-717.

36. Stöhr M, Dichgans J, Buettner UW, et al. Evozierte Potentiale. 3rd ed. Berlin: Springer-Verlag; 1996

37. Kulikowski JJ, Murray IJ. Occipital potentials evoked by chromatic and achromatic gratings in sedated macaques. J Pbysiol. 1988;399: 87.

38. Onofrj M, Gambi D, Bazzano S, et al. Evoked potentials (EPs) in experimental allergic encephalomyelitis: a study of EP modifications during the course of a controlled disease. Electromyogr Clin Neurophysiol. 1992;32:125-135.

39. Brok HPM., Bauer J, Jonker M, et al. Non-human primate models of multiple sclerosis. Immunol Rev. 2001;183:173-185. 